\title{
Bone Involvement in Hodgkin's Lymphoma: Clinical Features and Outcome
}

\author{
Francesco Gaudio $^{a}$ Pasquale Pedote ${ }^{b}$ Artor Niccoli Asabellac \\ Giuseppe Ingravallo ${ }^{d}$ Paola Sindaco ${ }^{a}$ Vito Alberotanza ${ }^{b}$ \\ Tommasina Perrone $^{a}$ Fiomena Emanuela Laddaga $^{d}$ Giuseppe Rubini ${ }^{c}$ \\ Amato Antonio Stabile lanora ${ }^{b}$ Giorgina Specchia ${ }^{a}$ \\ a Department of Emergency and Organ Transplantation (D.E.T.O.), Hematology Section, University of Bari, Bari, \\ Italy; ${ }^{b}$ Interdisciplinary Department of Medicine (DIM), Diagnostic Imaging Section, University of Bari, Bari, \\ Italy; ' Interdisciplinary Department of Medicine (DIM), Nuclear Medicine Section, University of Bari, Bari, Italy; \\ ${ }^{d}$ Department of Emergency and Organ Transplantation (D.E.T.O.), Pathology Section, University of Bari, Bari, Italy
}

\section{Keywords}

Extralymphatic · Bone $\cdot$ Hodgkin's lymphoma

\begin{abstract}
Hodgkin's lymphoma $(\mathrm{HL})$ is now a highly curable disease, with an improving 5-year survival rate that has now reached $86 \%$. At the time of presentation, $\mathrm{HL}$ is usually almost entirely confined to the lymph nodes. We performed a retrospective single-institution study of 384 cases with a median follow-up of 44 months, with the aim of identifying clinical and radiological characteristics and outcomes of patients with bone $\mathrm{HL} ; 32$ patients (8\%) had primary bone involvement, always with concurrent nodal disease. These included 22 men (69\%) and 10 women (31\%) with the median age as 41 years. Advanced stages and nodular sclerosis histology prevailed among the subgroup. Radiographic features of bone $\mathrm{HL}$ are not specific but indicate a destructive malignant
\end{abstract}

\section{KARGER}

(c) 2018 S. Karger AG, Basel

E-Mail karger@karger.com

www.karger.com/aha process with osteosclerosis and/or osteolysis. With current chemotherapeutic regimens, the long-term prognosis of patients with osseous HL appears good. The presence of bone lesions in $\mathrm{HL}$ should not be interpreted as implying a worse prognosis than without bone involvement.

(c) 2018 S. Karger AG, Basel

\section{Introduction}

Hodgkin's lymphoma (HL) accounts for $10-15 \%$ of lymphoma cases [1]. With current therapy, more than $75 \%$ of patients are likely to survive and remain diseasefree, for more than 5 years, and the great majority of these are invariably cured $[2,3]$. These results have been obtained, thanks to the judicious use of progressively improved staging methodologies over time, as well as the use of a stage-adapted treatment strategy [4-6]. 
At the time of presentation, HL is usually supra diaphragmatic, with a contiguous spread often occurring predictably from one nodal group to the next along the lymphatic pathways. HL is usually almost entirely confined to the lymph nodes. Extranodal lymphomas account for $2-16 \%$ of HL. Initial staging is crucial to demonstrate the presence of extra lymphatic involvement, which will affect therapeutic decision making $[7,8]$.

Lymphomas of the bone are uncommon, comprising only $8 \%$ of primary malignant bone tumors $[9,10]$.

Although radiologically detectable bone involvement is not unusual in advanced cases of HL, bone lesions are seldom the primary manifestations of this disease. HL involving osseous sites must also be distinguished from the involvement of the bone marrow by $\mathrm{HL}$, which occurs in approximately $5-10 \%$ of patients [11-13]. Unlike cases with bone marrow involvement, however, HL with clinically symptomatic bone involvement has been associated with similar or slightly better prognoses than those of patients with lymph node disease alone [14].

Radiographic evidence of bone involvement has been noted in $10-15 \%$ of cases of HL; the radiologic features of bone HL have been described as osteosclerotic, osteolytic, or mixed osteosclerotic/osteolytic [15-18].

We present a series of 32 cases of bone HL from the clinical, radiologic, and pathologic perspectives. The aim of this article is to summarize the main features and to define the prognostic impact of HL in patients with bone involvement treated at our center.

\section{Patients}

This retrospective study was focused on 384 patients with newly diagnosed classic HL treated at Bari University Hospital (Italy) between January 2006 and June 2017. Histologic diagnoses were established according to the WHO classification.

Patients diagnosed with nodular lymphocyte-predominant HL, those who were human immunodeficiency virus (HIV) positive, and those treated only with radiation or palliative care were excluded.

Informed consent was obtained from all patients for inclusion in the study. No patient refused authorization to use their medical records for research. No patient was lost to follow-up.

We performed a retrospective single-institution study of 384 cases (234 [61\%] males and 150 [39\%] females) with a median follow-up of 44 months. The median age at diagnosis was 36 years (range 15-83), 119 patients (31\%) had advanced stage disease (IIIIV), 145 (38\%) bulky disease, 180 (47\%) presented B symptoms, and $103(27 \%)$ had spleen involvement. The histology was nodular sclerosis in $322(84 \%)$; in $73(19 \%)$, the histology was extralymphatic disease. All the patients were treated with the ABVD regi- men; 269 patients $(70 \%)$ received radiotherapy of the involved field. All patients underwent biopsy to confirm the bone involvement.

\section{Staging and Routine Laboratory Evaluations}

All patients were clinically staged according to the Ann-Arbor system, including medical history, complete physical examination, blood counts, biochemical profile, chest X-ray films, computed tomography of the chest, abdomen and pelvis, Fluorodeoxyglucose positive emission tomography (PET/CT) total body, and unilateral bone marrow biopsy. Hemoglobin concentrations, white blood cell counts and differential, erythrocyte sedimentation rate, serum albumin and serum lactate dehydrogenase levels were measured by standard assays. Anemia was defined as the presence of hemoglobin levels $<13 \mathrm{~g} / \mathrm{dL}$ for males and $<11.5 \mathrm{~g} / \mathrm{dL}$ for females. Serum albumin was analyzed with a cutoff value of $3.5 \mathrm{~g} / \mathrm{dL}$, which is the normal lower limit at our laboratory. Serum b2-microglobulin was measured by radioimmunoassay (normal values: $1.0-2.4 \mathrm{mg} / \mathrm{L}$ ).

\section{Response Evaluation}

Tumor responses were assessed at the end of the treatment and were classified as complete response, partial response, stable disease, or progressive disease according to the International Workshop Criteria.

Definitions of response criteria and progression-free survival (PFS) were based on the International Harmonization Project Lymphoma guidelines. PFS was defined as the time from $\mathrm{cHL}$ diagnosis to the time to progression, relapse from complete response, death as a result of any cause, or last follow-up [19].

\section{Statistical Analysis}

The estimation of actuarial PFS was performed by the KaplanMeier method. The identification of prognostic factors at univariate analysis was based on the log-rank test.

Chi-square test was used to assess the main differences between clinical features of the bone involvement group and of the other patients.

\section{Results}

During the study period, 384 newly diagnosed HL cases were treated with the ABVD regimen.

\section{Extralymphatic Disease and Bone Involvement}

Extralymphatic disease was diagnosed in 73 patients (19\%). The sites were the lung in $31(42 \%)$, bone in 32 $(43 \%)$, liver in $6(8 \%)$, and kidney in $3(4 \%)$.

Of the 384 eligible patients, 32 had primary bone involvement, with concurrent nodal disease. These included 22 men and 10 women with a median age of 41 years. Advanced stages and nodular sclerosis histology prevailed among the subgroup; Table 1 summarizes the basic characteristics of patients with bone involvement. Eight patients presented with pain of the involved area, while the others were asymptomatic. 
Table 1. Patients characteristics

\begin{tabular}{lcc}
\hline Total $(n=32)$ & $n$ & $\%$ \\
\hline Age, years, median (range) & $41(18-79)$ & \\
Gender & & \\
$\quad$ Male & 22 & 68 \\
$\quad$ Female & 10 & 32 \\
Histology & & \\
$\quad$ Scleronodular & 31 & 97 \\
$\quad$ Mixed cellularity & 1 & 3 \\
Stage & 1 & 3 \\
$\quad$ I-II & 4 & 12 \\
$\quad$ III & 27 & 85 \\
$\quad$ IV & 18 & 56 \\
B symptoms & 26 & 81 \\
High ESR & 12 & 37 \\
Bulky disease & 3 & 9 \\
Spleen involvement & & \\
\hline
\end{tabular}

Table 2. CT imaging

\begin{tabular}{lrr}
\hline & $n$ & $\%$ \\
\hline CT-detectable lesions & 20 & \\
$\quad$ Yes & 12 & 63 \\
No & & 37 \\
CT imaging of lesions & 12 & 38 \\
Osteolytic & 3 & 9 \\
Osteosclerotic & 7 & 22 \\
Mixed & 10 & 31 \\
Not detected & & \\
\hline
\end{tabular}

\section{Radiologic Features}

Radiographs of the patients were available for review; features are shown in Table 2 and Table 3.

Studies included CT, PET/CT scans, and magnetic resonance imaging (MRI). Lesions were located in the axial and proximal appendicular skeleton; 51 were axial. The 4 appendicular lesions were present in the proximal regions of long bones.

The radiographic features of osseous HL were variable, featuring osteolysis in $34 \%$ of cases, osteosclerosis in $6 \%$, and a mixture of osteolysis and osteosclerosis in $22 \%$. Lesional margins were ill-defined, and there was frequent cortical destruction, periosteal reaction, and tumor extension into surrounding soft tissue. The involvement was most frequently found on CT scan and confirmed by PET/CT scan and MRI. In 12 patients, CT scan was negative and the bone involvement was diagnosed exclusively by PET/CT scan. CT was beneficial for assessing periosteal reactions and cortical destruction. The radiographic differential diagnosis included primary sarcomas of the bone, non- Hodgkin lymphomas and leukemia, osteomyelitis, and metastatic lesions.

\section{Pathologic Features}

Histologically, osseous HL was typically present as an infiltrate between intact bone trabeculae, similar to nonHodgkin lymphomas involving the bone. In some cases, bone formation was noted in cortical regions or around bone trabeculae, corresponding to periosteal reactions and osteosclerosis noted on radiographs. The infiltrate consisted of lymphocytes, eosinophils, plasma cells, and histiocytes, with atypical mononuclear cells and ReedSternberg cells. Well-formed granulomas, such as those present in fungal or mycobacterial infections, or sarcoidosis, were not present in any case. Immunohistochemical stains for CD15 and CD30 were positive in neoplastic cells, with corresponding nonimmunoreactivity to B-and T-cell markers.

\section{Survival Analysis}

All patients were treated with the ABVD chemotherapy regimen; 12 of them also underwent irradiation because they had intermediate stage disease (where radiotherapy is a standard part of the curative treatment) or residual disease after chemotherapy in advanced stage disease. The bone sites involved were included as a part of the radiotherapy target volume in 4 cases.

PFS in the 3 groups - bone, lymphatic and extralymphatic HL - is shown in Figure 1; at 3 years PFS was 81, 78 and $51 \%$ in these groups respectively $(p<0.05)$. Survival was significantly reduced in patients with extralymphatic disease other than the bone subgroup but not for those with bone disease.

\section{Discussion}

An accurate assessment of the stage of disease in patients with HL is critical for the selection of the appropriate therapy [1-3]. The staging system for patients with HL is based on whether the involved lymph nodes are on one or both sides of the diaphragm, whether the number of involved sites of involvement are bulky, whether there is contiguous extranodal involvement or disseminated extranodal disease, and whether typical systemic symptoms (B symptoms) are present. PET/CT scanning has emerged as an important tool in the staging of patients with HL, and this adds significantly to the staging information obtained using other standard radiographic methods $[4,5]$. 
Table 3. Bone lesions features

\begin{tabular}{lrr}
\hline & $n$ & $\%$ \\
\hline Bone involvement by site & & \\
$\quad$ Vertebral & 20 & 36 \\
$\quad$ Pelvis & 18 & 33 \\
Sternum & 9 & 16 \\
Ribs & 3 & 6 \\
Limbs & 4 & 7 \\
Skull & 1 & 2 \\
Number of bone lesions & & \\
$\quad$ Multiple & 15 & 47 \\
Single & 14 & 44 \\
$\quad$ Extension to soft tissue & 3 & 9 \\
\hline
\end{tabular}

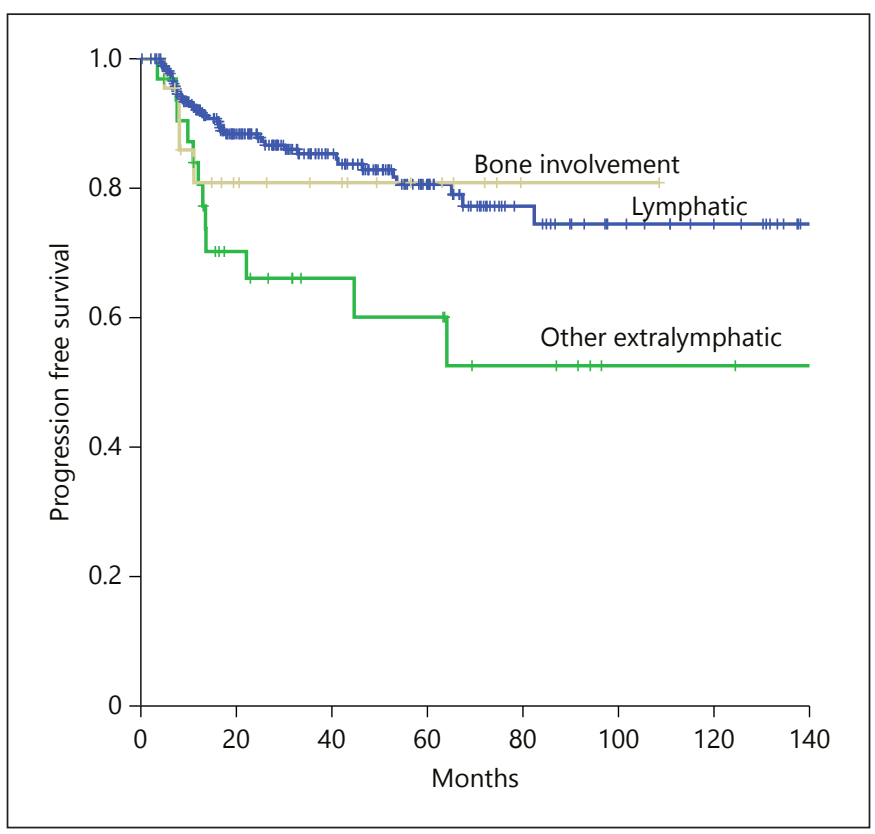

Fig. 1. Progression-free survival by disease site (log-rank test- $p<$ 0.05).

In the diagnosis and management of HL, 18F-fluorodeoxyglucose PET/CT is a functional imaging modality that has become a standard tool complementing contrastenhanced CT scans [9-12]. Several studies have shown that PET/CT helps to make a more accurate identification of the correct pretreatment stage in HL compared with contrast-enhanced CT, although PET/CT upstages disease from an early to an advanced stage in only $10-15 \%$ of patients whose treatment ultimately undergoes modification $[4,5]$.

Extranodal involvement is much less common in HL than in non-HL [8-10]. In this retrospective study of 384 newly diagnosed HL cases, extralymphatic disease was diagnosed in 19\% of cases. The most commonly affected sites were the lung in $42 \%$ of cases and bone in $44 \%$. Bone involvement is considered a rare extralymphatic spread. In the literature, a wide range of incidence, from 0.1 to $15 \%$, is reported [14-18]. Clinical pictures of osseous HL are not specific; some patients have pain as the first symptom, while some others are asymptomatic and the diagnosis is coincidental. The lesions have no uniform appearance - they may be osteolytic, osteosclerotic, or mixed $[15,16]$.

Histological diagnosis from the bone biopsy specimen is very difficult, since necrosis and polymorphonuclear cell infiltration are often present. The increasing use of $\mathrm{PET} / \mathrm{CT}$ scan in the staging may help to solve some differential diagnosis problems. At the present time, we diagnose osseous HL involvement in advanced stage patients with lesion(s) in the axial skeleton found on CT scan. In other patients, we recommend further diagnostic procedures such as MRI or PET/CT; we do not perform routine biopsy of the lesions. Furthermore, although regional lymph nodes may be involved in cases of osseous HL, less than half of the cases in our study presenting with both osseous and nonosseous disease had lymph node disease that could be classified as contiguous with osseous foci. Although osseous HL presenting in the sternum, sometimes with an associated soft tissue mass, has been reported as an unusual presentation of this tumor, 3 of our cases had sternal involvement. Lytic lesions were sometimes interpreted as representative of hematogenous spread, and sclerotic lesions as direct extensions of disease from contiguous lymph nodes [16-18].

Bone involvement alone has not proven to be an adverse prognostic factor. Because of the frequent multiplicity of osseous lesions, in advanced stage disease, strong co-variations are observed, and constitute an adverse prognostic factor. The outcome of patients with osseous HL has been reported not to be worse than that of patients without bone involvement, and this was true for our patients as well. At present, therefore, we may conclude that there is no need for a special treatment strategy for osseous HL. The response rate was somewhat lower in our patients with bone involvement, but the number of patients is low and insufficient to draw any definitive conclusion. However, the evaluation of treatment response is generally problematic. Bones regenerate very slowly and morphological changes in the bone structure often persist after treatment. It is sometimes impossible to discriminate between complete and partial remission. The sensitivity and specificity of functional 
imaging for bone involvement have not yet been established and there are high numbers of false positive results due to benign muscular hypermetabolism; false positivity has been described in bone marrow as well [20] Whether additional prophylactic radiotherapy of the initially involved bone sites is advisable is a question that still has to be answered.

In conclusion, bones are relatively common extralymphatic sites of HL. Diagnosis can be difficult and standard staging and restaging procedures have not yet been determined. Treatment strategies and the prognosis depend on the stage of the disease, but there does not appear to be a need for additional treatment.

\section{Disclosure Statement}

The authors declare that they have no conflicts of interest to disclose.

\section{References}

1 Harris NL: Hodgkin's lymphomas: classification, diagnosis, and grading. Semin Hematol 1999;36:220-232.

2 Canellos GP, Rosenberg SA, Friedberg JW, Lister TA, Devita VT: Treatment of Hodgkin lymphoma: a 50-year perspective. J Clin Oncol 2014;32:163-168.

3 Gaudio F, Giordano A, Pavone V, Perrone T, Curci P, Pastore D, Delia M, De' Risi C, Spina A, Liso V, Specchia G: Outcome of very late relapse in patients with Hodgkin's lymphomas. Adv Hematol 2011;2011:707542.

4 Kostakoglu L, Evens AM: FDG-PET Imaging for Hodgkin lymphoma: current use and future applications. Clin Adv Hematol Oncol 2014;12:20-35

5 Hutchings $M$, Loft $A$, Hansen M, Pedersen LM, Berthelsen AK, Keiding S, D’Amore F, Boesen AM, Roemer L, Specht L: Position emission tomography with or without computed tomography in the primary staging of Hodgkin's lymphoma. Haematologica 2006; 91:482-489.

6 Bartlett NL: Limited-stage Hodgkin lymphoma: optimal chemotherapy and the role of radiotherapy. Am Soc Clin Oncol Educ Book 2013;374-380.

7 Gaudio F, Pedote P, Asabella AN, Perrone T, Laddaga FE, Sindaco P, Cimmino A, D’Abbicco D, Pezzolla A, Rubini G, Specchia G: Extralymphatic disease is an independent prognostic factor in Hodgkin lymphoma.
Clin Lymphoma Myeloma Leuk 2018;18: e261-e266.

8 Ömür O, Baran Y, Oral A, Ceylan Y: Fluorine-18 fluorodeoxyglucose PET-CT for extranodal staging of non-Hodgkin and Hodgkin lymphoma. Diagn Interv Radiol 2014 20:185-192.

9 Gebert C, Hardes J, Ahrens H, Buerger H, Winkelmann W, Gosheger G: Primary multifocal osseous Hodgkin disease: a case report and review of the literature. J Cancer Res Clin Oncol 2005;131:163-168.

10 Baar J, Burkes RL, Bell R, Blackstein ME, Fernandes B, Langer F: Primary non-Hodgkin's lymphoma of bone. Cancer 1994;73:11941199.

11 Bartl R, Frisch B, Burkhardt R, Huhn D, Pappenberger R: Assessment of bone marrow histology in Hodgkin's disease: correlation with clinical factors. Br J Haematol 1982;51:345360.

12 Lambertenghi-Deliliers G1, Annaloro C, Soligo D, Oriani A, Pozzoli E, Quirici N, Luksch R, Polli EE: Incidence and histological features of bone marrow involvement in malignant lymphomas. Ann Hematol 1992;65:61-65.

13 Rosenberg SA: Hodgkin's disease of the bone marrow. Cancer Res 1971;31:1733-1736.

14 Stuhlbarg J, Ellis FW: Hodgkin's disease of bone: favorable prognostic significance? Am J Roentgenol Radium Ther Nucl Med 1965; 93:568-572.
15 Edeiken-Monroe B, Edeiken J, Kim EE: Radiologic concepts of lymphoma of bone. Radiol Clin North Am 1990;28:841-864.

16 Braunstein EM: Hodgkin's disease of bone: radiographic correlation with the histological classification. Radiology 1980;137:643-646.

17 Pear BL: Skeletal manifestations of lymphoma and leukemias. Semin Roentgenol 1974;9: 229-240.

18 Ozdemirli M, Mankin HJ, Aisenberg AC, Harris NL: Hodgkin's disease presenting as a solitary bone tumor. Cancer 1996;77:79-88.

19 Cheson BD, Pfistner B, Juweid ME, Gascoyne RD, Specht L, Horning SJ, Coiffier B, Fisher RI, Hagenbeek A, Zucca E, Rosen ST, Stroobants S, Lister TA, Hoppe RT, Dreyling M, Tobinai K, Vose JM, Connors JM, Federico M, Diehl V: International Harmonization Project on Lymphoma Revised response criteria for malignant lymphoma. J Clin Oncol 2007;25:579-586.

20 Gallamini A, Hutchings M, Rigacci L, Specht L, Merli F, Hansen M, Patti C, Loft A, Di Raimondo F, D’Amore F, Biggi A, Vitolo U, Stelitano C, Sancetta R, Trentin L, Luminari S, Iannitto E, Viviani S, Pierri I, Levis A: Early interim 2-[18F]fluoro-2-deoxy-D-glucose positron emission tomography is prognostically superior to international prognostic score in advanced-stage Hodgkin's lymphoma: a report from a joint Italian-Danish study. J Clin Oncol 2007;25:3746-3752. 